
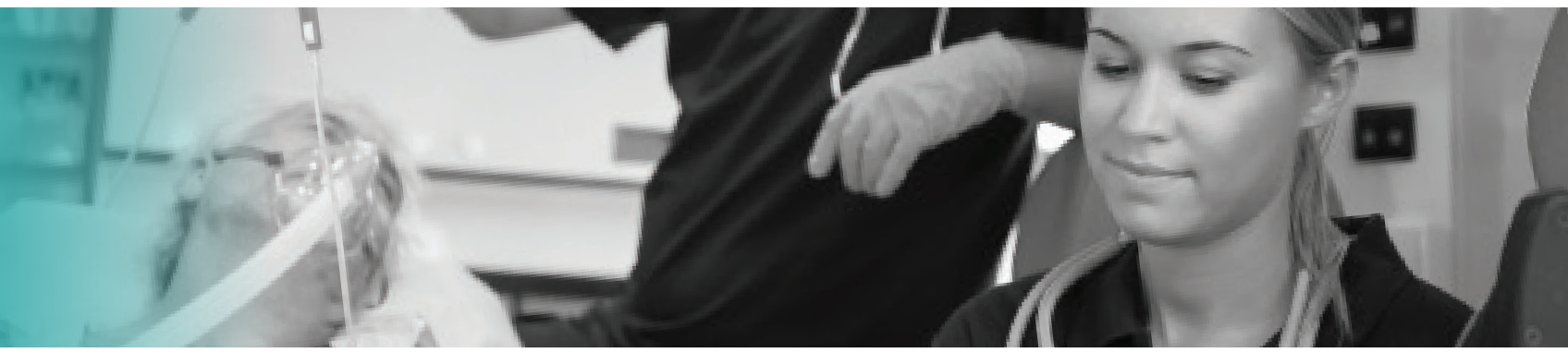

Volume 15 | Issue 1 | Article 1
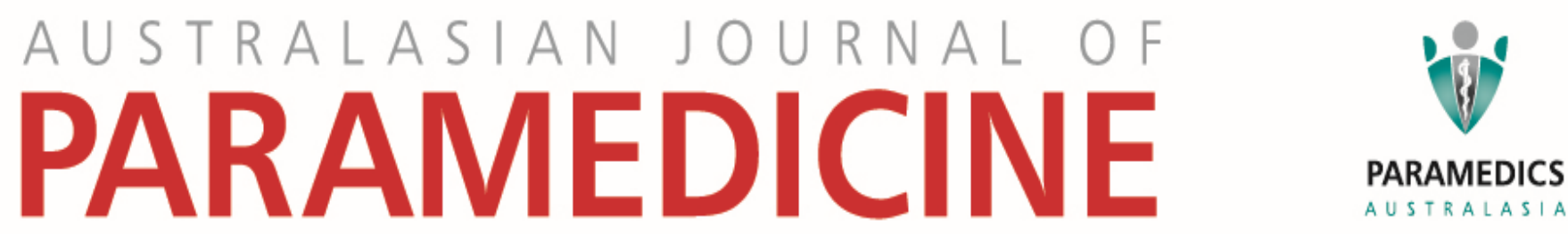

Improving paramedic confidence with paediatric patients: A scoping review

James Fowler

Monash University, Melbourne, Victoria

Bronwyn Beovich

Monash University, Melbourne, Victoria

Brett Williams

Monash University, Melbourne, Victoria 


\title{
Improving paramedic confidence with paediatric patients: A scoping review
}

\author{
James Fowler BNurs, BEmergHlth(PMed) is Research Assistant ${ }^{1}$, Bronwyn Beovich DipAppSc(Nsg), BAppSc(Ch),
} MChSc is Research Assistant ${ }^{1}$, Brett Williams PhD is Head of Department ${ }^{1}$

\author{
Affiliations: \\ ${ }^{1}$ Department of Community Emergency Health and Paramedic Practice, Monash University, Melbourne, Victoria
}

\section{Abstract}

\section{Introduction}

Paediatric patients comprise a reasonable minority of paramedic workload, but cases involving children often evoke anxiety and discomfort. Subsequent feelings of low confidence and self-efficacy have been linked with a reluctance to initiate treatment and possibly, poorer care. It is unknown to what extent these apprehension-producing perceptions are held by paramedics and what educational strategies can improve them. This study aims to fill this gap by reporting on a scoping review that examined and mapped the effectiveness of educational interventions on improving paramedic perceptions of caring for paediatric patients.

\section{Methods}

Arksey and O'Malley's scoping methodology was used. CINAHL, AMED, EMBASE, MEDLINE and PsychINFO databases were searched, alongside several networks and grey literature services. The search strategy was validated by a subject researchlibrarian. Two reviewers independently reviewed retrieved articles against inclusion and exclusion criteria determined in advance.

\section{Results}

Seventeen articles were included in the review, comprising six conference abstracts, one letter to the editor and 10 journal articles. These articles were categorised into three themes: identifying educational needs and preferences, novel education programs and evaluating education delivery methods.

\section{Conclusion}

Paramedics feel uncomfortable treating paediatric patients and desire more paediatric training, particularly simulation and exposure to children. There is insufficient evidence to demonstrate superior effectiveness of any particular training program. While the most effective education delivery method is unclear, it seems web-based learning can be as effective as traditional teaching. The efficacy of existing pre-established paediatric training programs needs to be investigated, alongside paediatric education in the initial training curriculum. Further research, particularly from outside the United States of America, is needed to identify and develop the most effective educational intervention to improve paramedic confidence with paediatric patients.

Keywords:

pediatrics; pre-hospital care; emotions; self efficacy; education

Corresponding author: James Fowler, james.fowler01@gmail.com 


\section{Introduction}

Paramedics are often the first responders to cases of paediatric trauma or acute illness. It is therefore vital that they have the appropriate knowledge, skills and confidence to render effective assistance. Paediatric patients make up 4.5$13 \%$ of total ambulance calls (1-5); with trauma being a major cause of child mortality (6-8). Observational and anecdotal evidence suggests that the pre-hospital management of paediatric patients is inherently stressful, causing anxiety and discomfort for many paramedics (9-12). Although only a small proportion of paediatric cases require emergency intervention due to a life-threatening condition (4), children still present unique challenges to emergency care professionals as they are anatomically, physiologically and developmentally different to adults (13).

As a minority of pre-hospital clinical work involves a paediatric emergency it can be challenging to maintain confidence with limited opportunities for clinical practice. In Australia, it has been estimated that a paramedic will attend a paediatric cardiac arrest only once every 12.5 years (14) and as such, paediatric pre-hospital emergency management is often viewed as a 'low frequency, high risk event' by paramedics (15). Despite this, there is a general view that adequate paediatric education is lacking, both in initial education as well as in ongoing professional development (16-18). A recent paper highlights that minimum paediatric education standards for pre-hospital providers have been proposed for initial and ongoing certification in the United States of America (USA), however, it is not mandatory to adopt them and as such standards are highly variable (19). In Australia, the majority of paramedic education is undertaken within various university settings, however several vocational courses remain an option for those wishing to work as a paramedic (20). It is therefore reasonable to assume that there may be considerable variation in paediatric knowledge and skill within the workforce.

The identified lack of paediatric training and experience along with the anxiety and discomfort of caring for this patient group may be linked with pre-hospital treatment errors (11). This can involve errors of commission such as administering an incorrect drug dose, or more commonly errors of omission such as reluctance to initiate required treatment in a timely manner (11). Some paramedics may prefer to quickly transport the patient to a well-resourced emergency department ('scoop and run') in preference to stabilising the child at the scene (12). It is possible that such situations contribute adversely to patient safety $(12,21)$ and are at odds with the developing professional identity of paramedics as advanced clinical decision-makers (21-23).

It is largely unknown what educational interventions have a positive effect on paramedic confidence and self-efficacy when providing care for paediatric patients. A preliminary search revealed no existing reviews relating to this concept. Scoping reviews allow mapping of the key concepts and the evidence supporting a research domain of interest (24-26). Compared to systematic reviews with strict criteria for study inclusion, scoping reviews incorporate a wider range of research materials that are both peer-reviewed and nonpeer-reviewed in order to provide a broader understanding of the topic $(24,25)$. Considered alongside the breadth of the concept of interest, exploration of literature surrounding this topic therefore lends itself to a scoping review. The objective of this study was to fill the aforementioned identified gap in the literature by performing a scoping review to examine and map the effectiveness of educational interventions on improving paramedic perceptions of providing care for paediatric patients.

\section{Methods}

Arksey and O'Malley's (25) six-stage scoping methodology was used as the framework for this scoping review, alongside consideration of enhancements suggested by Levac, Colquhoun and O'Brien (27) and the Joanna Briggs Institute methodology for scoping reviews (28). The six stages described by Arksey and O'Malley (25) are:

1. Identifying the research question

2. Identifying relevant studies

3. Study selection

4. Charting the data

5. Collating, summarising and reporting the results

6. Consultation (optional).

Each of these stages is outlined below.

\section{Identifying the research question}

The research question guiding this scoping review was 'What educational interventions improve paramedic confidence when dealing with paediatric patients?'

\section{Identify relevant studies}

Preliminary searches of online databases CINAHL and EMBASE were performed to enable familiarisation with keywords and to aid in the development of the final search strategy. Following advice from a subject research-librarian, search terms were clustered under four search strategy components that were later combined to produce the full strategy:

1. Paramedic component, for which a previously developed pre-hospital search filter was used (29)

2. Paediatric component

3. Educational interventions component

4. Confidence component. 
The full search strategy was ratified by a subject research librarian and is included in Appendix 1. This strategy was used to search five online databases in October 2015: CINAHL, AMED, EMBASE, MEDLINE and PsychINFO. Google Scholar, the grey literature site greylit.org and seven relevant professional networks were also searched using a modified search strategy based on the aforementioned search components. There were no restrictions on dates of publication. The bibliographies of articles that were included following the title and abstract screen were also reviewed, producing no new articles.

\section{Study selection}

Articles retrieved from stage two were screened against a documented inclusion and exclusion criteria determined in advance (Appendix 2). Following the Joanna Briggs Institute methodology (28), the inclusion criteria were divided into three components: population, concept and context.

One author screened 2001 retrieved articles by title and abstract, yielding 34 included articles. A further full text screen was performed by two authors, yielding 17 articles included in the final review. Reasons for exclusion at the full text screening stage were recorded by both reviewers. Common reasons included non-paramedic population and/or context and educational interventions not addressing paramedic confidence. Figure 1 summarises the study selection process.

\section{Charting the data}

The following information was extracted from all included articles and recorded in a table: author(s), date of publication, study location, study purpose, study methodology and relevant results. Table 1 provides this overview of included articles.

Figure 1. Stage three: article selection

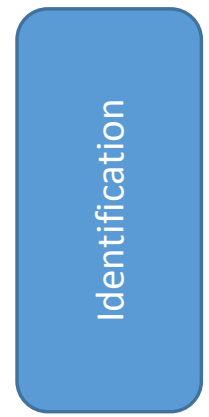

$$
\begin{gathered}
\text { Articles identified through } \\
\text { database searching } \\
(\mathrm{n}=2594)
\end{gathered}
$$
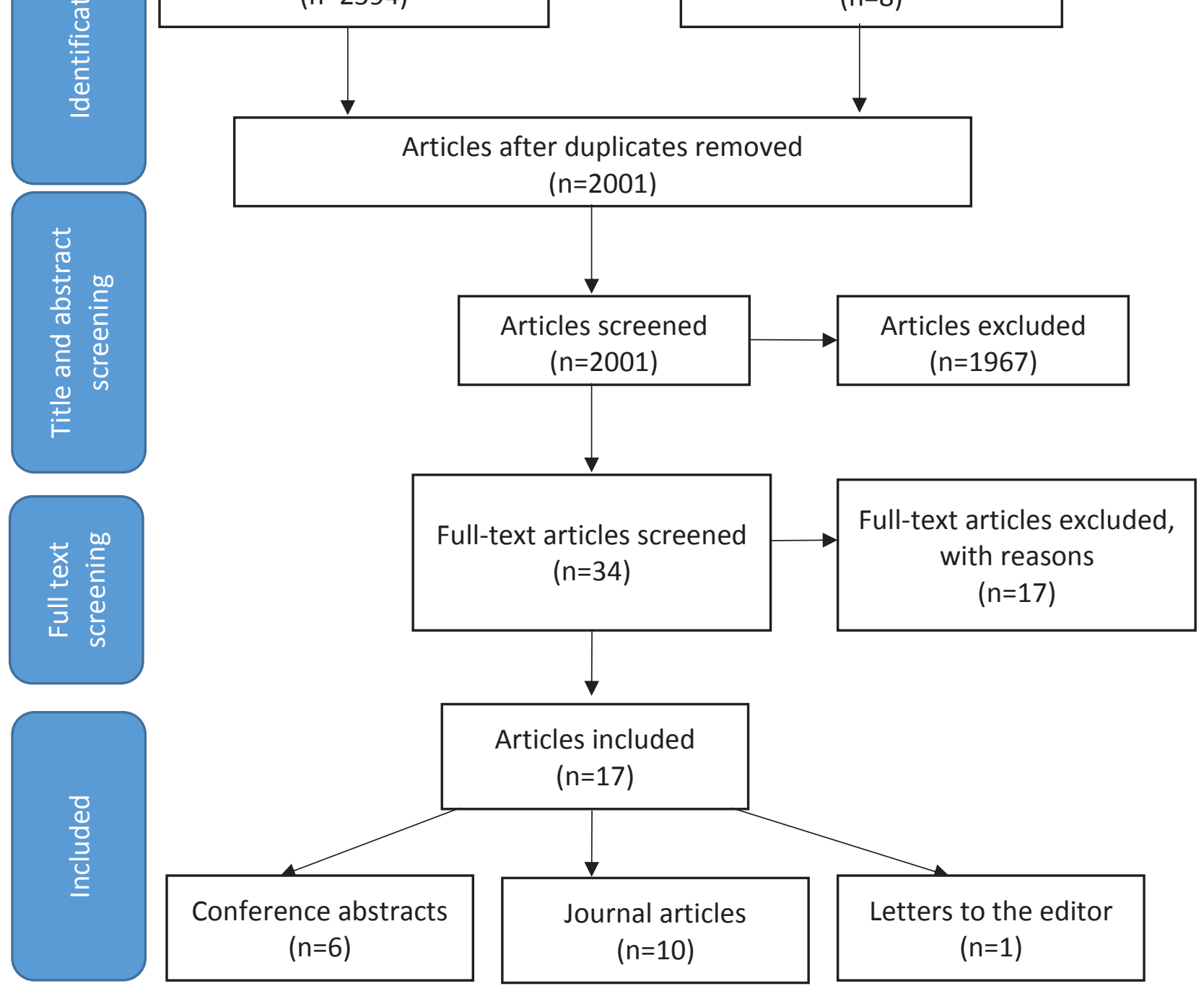

Articles included

$$
(n=17)
$$

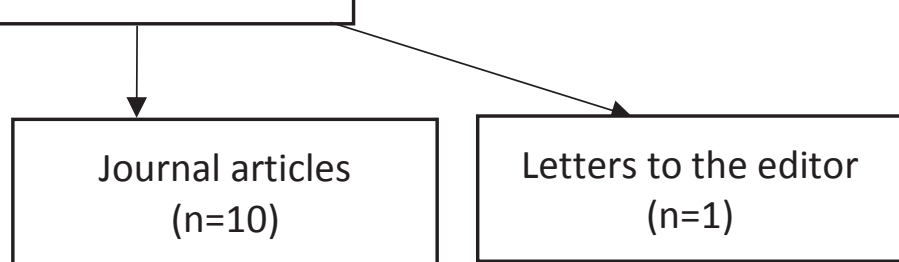




\begin{tabular}{|c|c|c|c|c|c|c|}
\hline 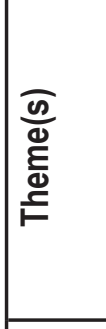 & 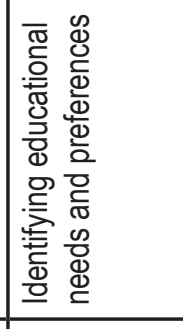 & 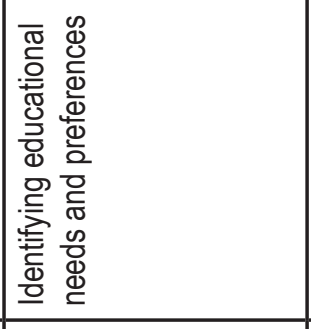 & 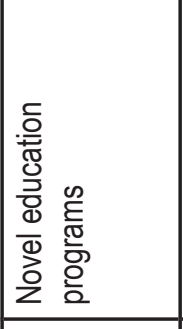 & 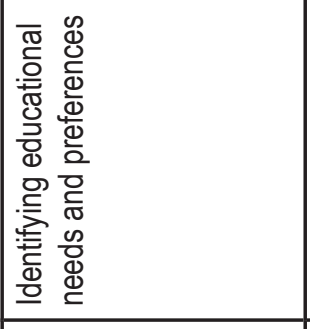 & 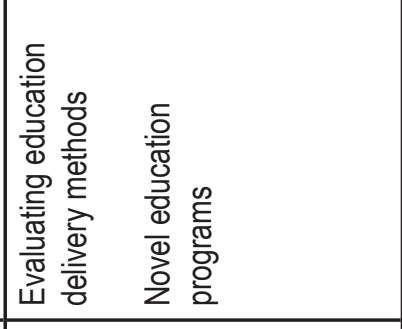 & 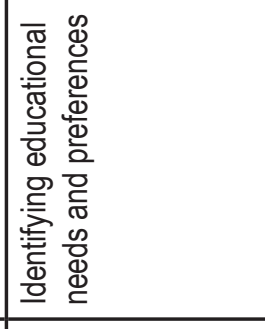 \\
\hline & 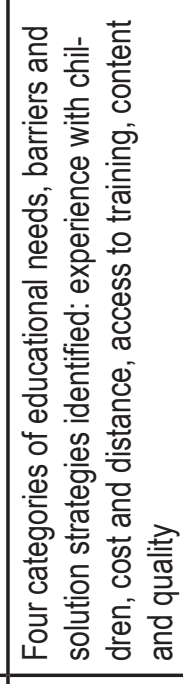 & 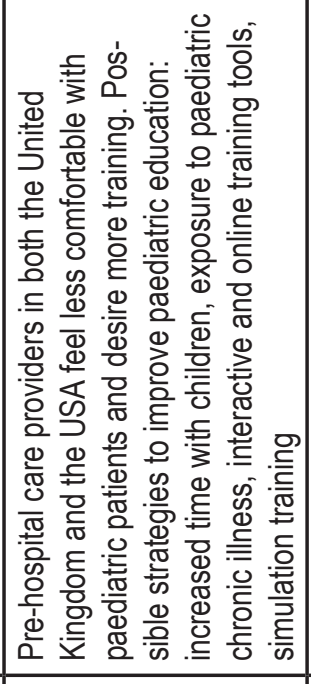 & 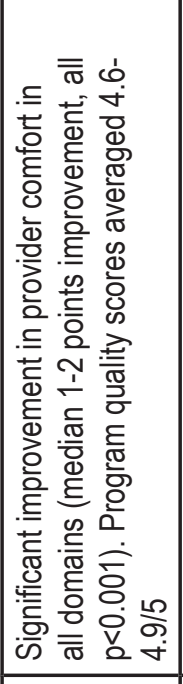 & 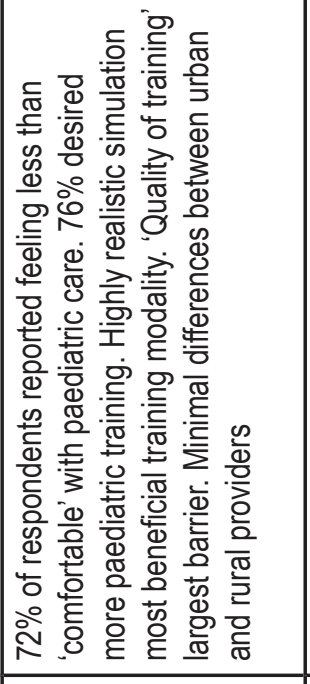 & 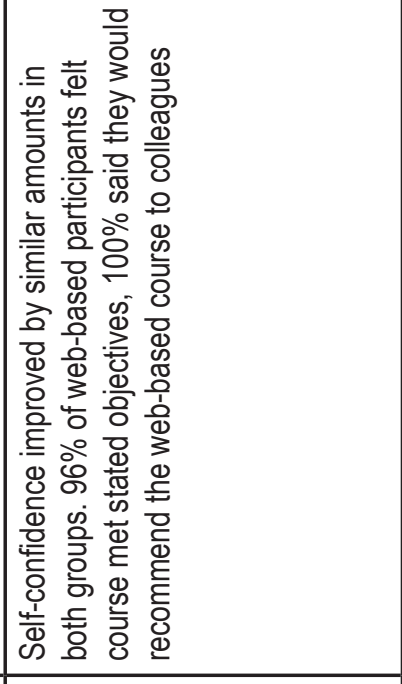 & 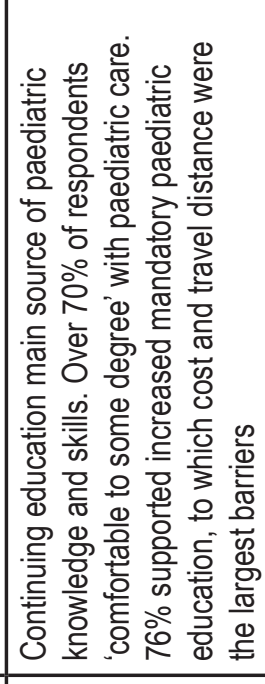 \\
\hline 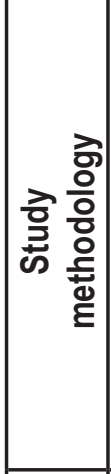 & 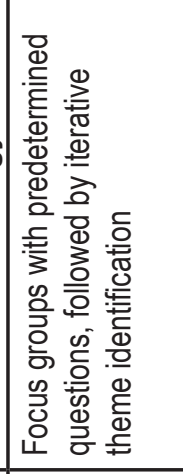 & 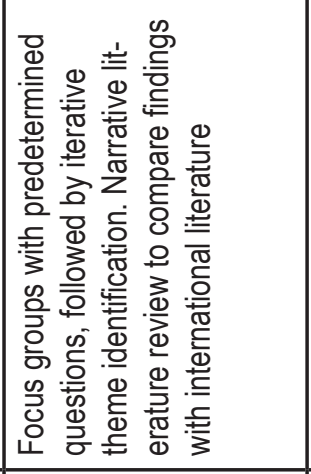 & 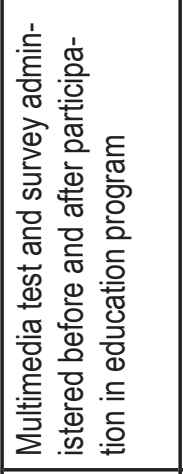 & 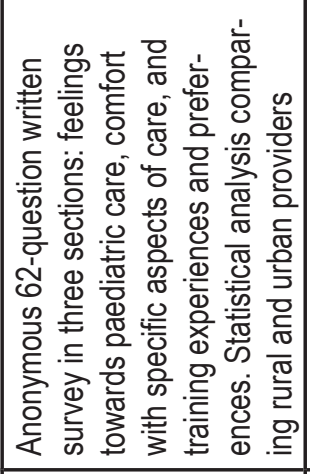 & 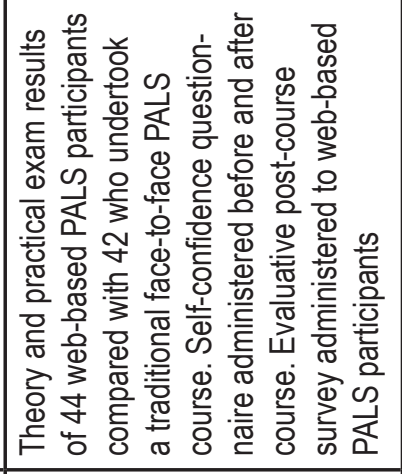 & 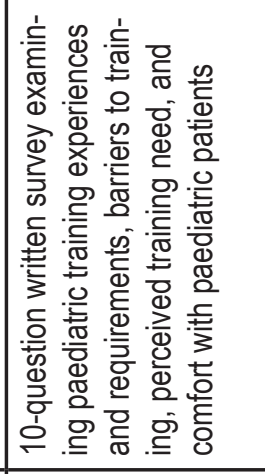 \\
\hline 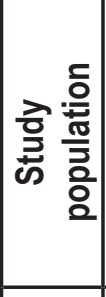 & 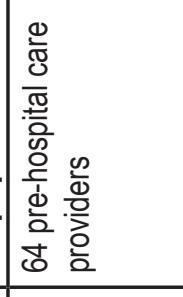 & 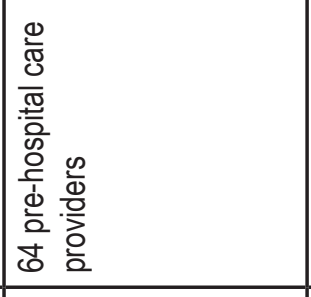 & 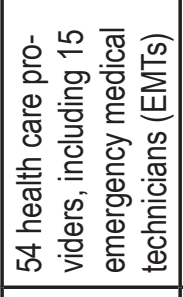 & 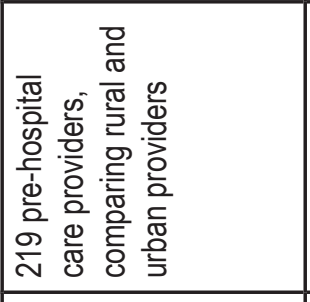 & 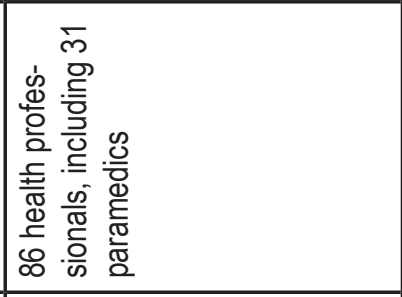 & 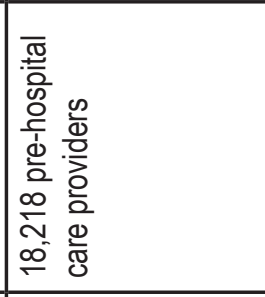 \\
\hline 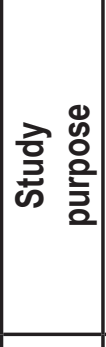 & 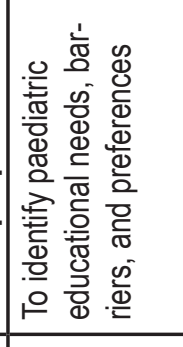 & 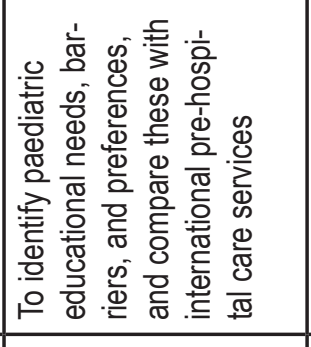 & 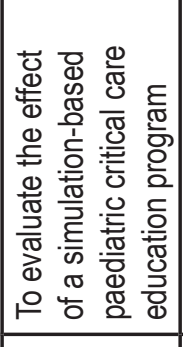 & 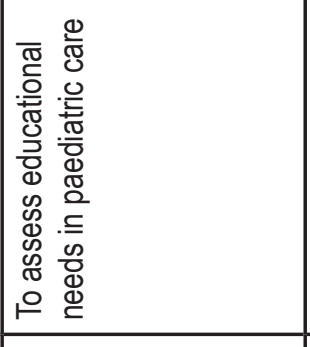 & 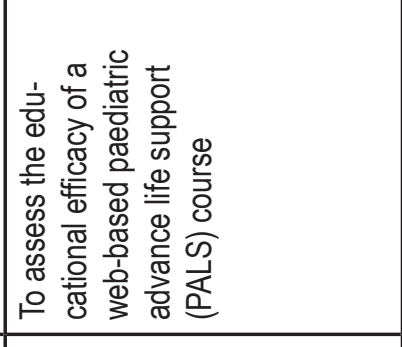 & 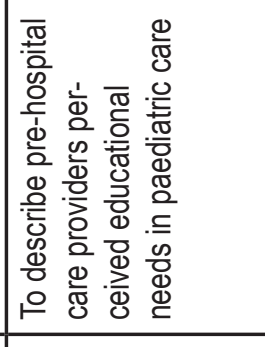 \\
\hline 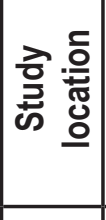 & 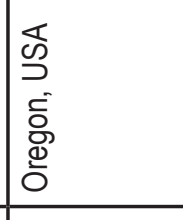 & 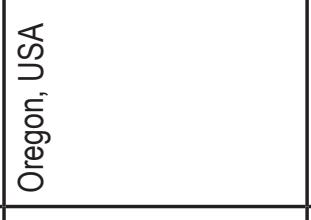 & 芯 & 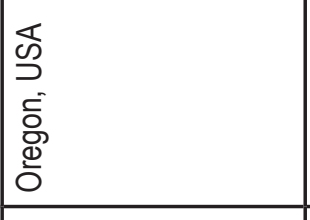 & 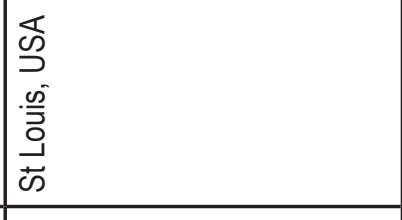 & 胨 \\
\hline 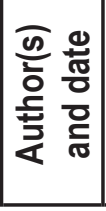 & 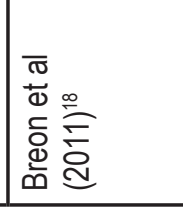 & 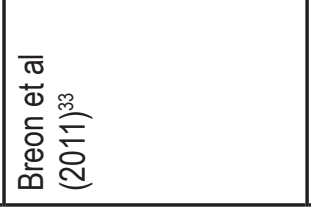 & 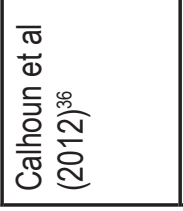 & 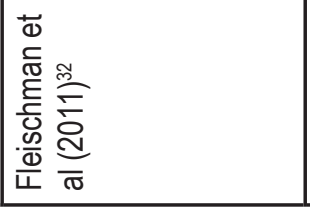 & 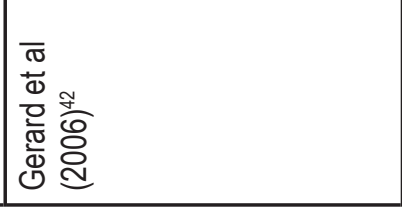 & 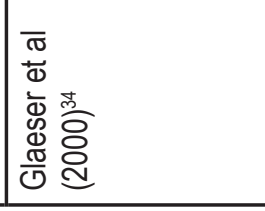 \\
\hline
\end{tabular}




\begin{tabular}{|c|c|c|c|c|c|c|}
\hline 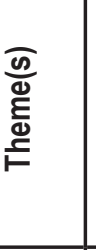 & 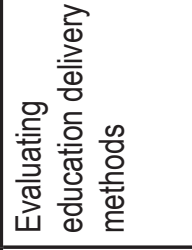 & 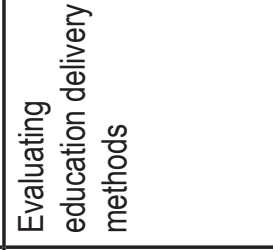 & 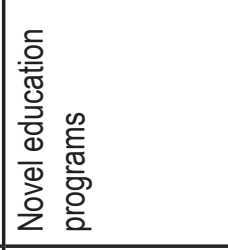 & 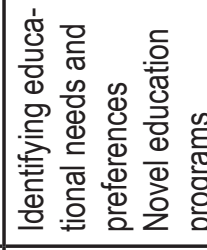 & 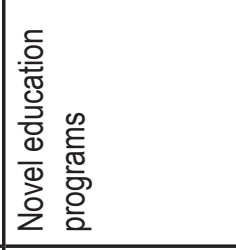 & 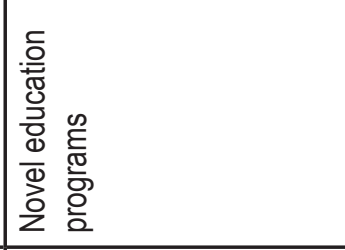 \\
\hline 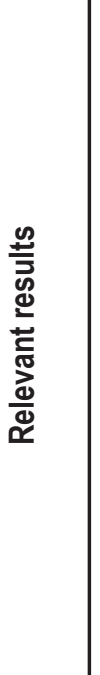 & 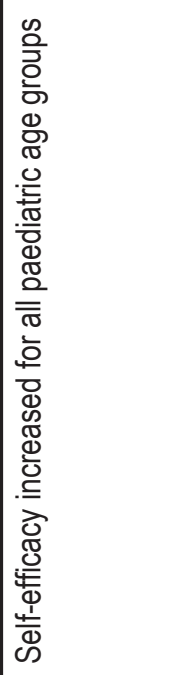 & 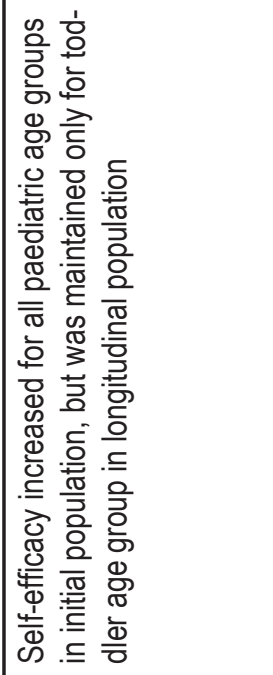 & 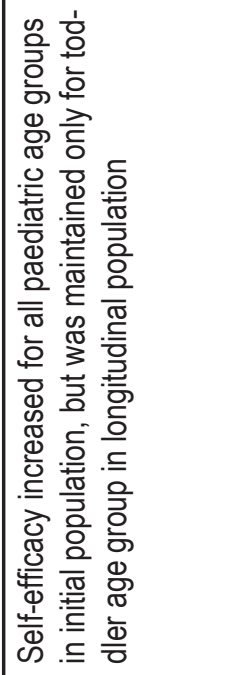 & 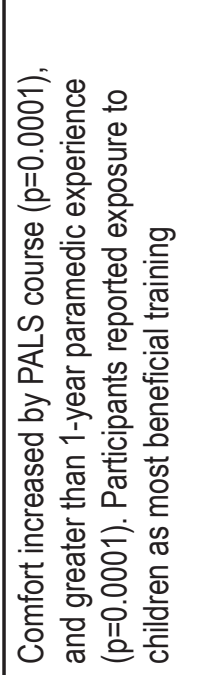 & 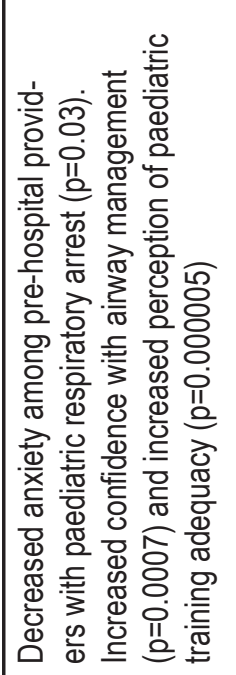 & 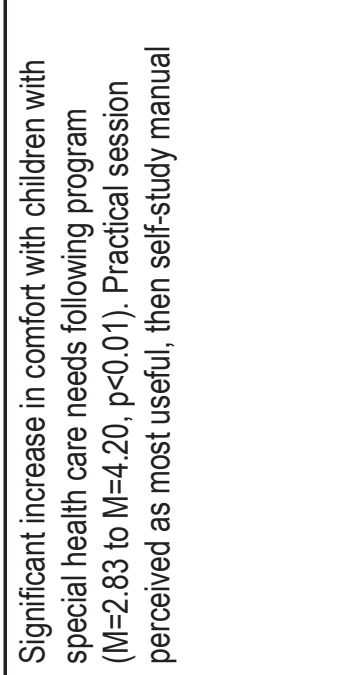 \\
\hline 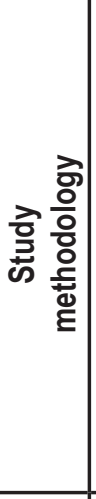 & 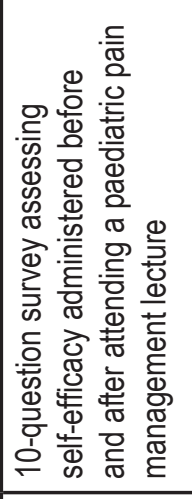 & 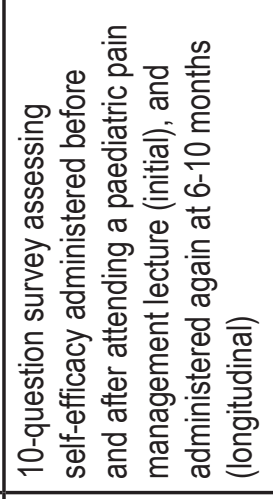 & 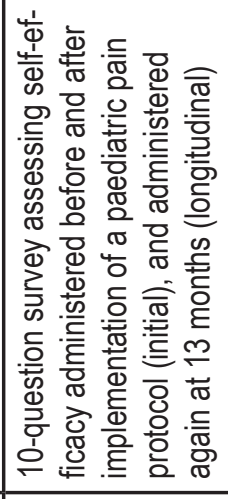 & 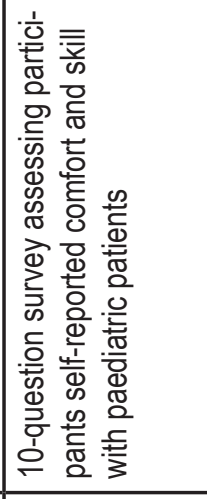 & 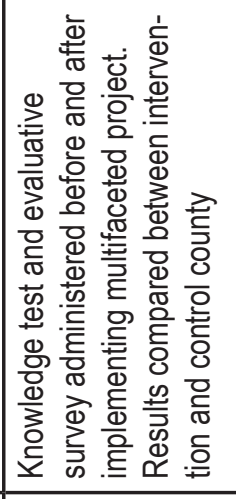 & 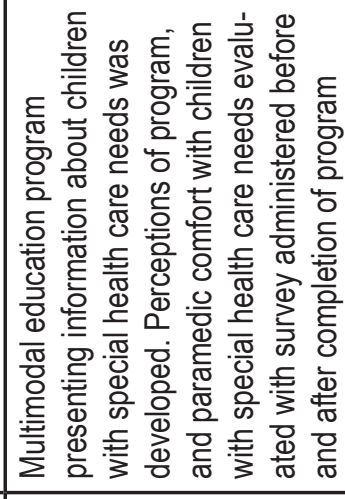 \\
\hline 흘 & 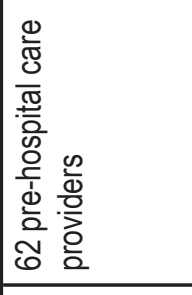 & 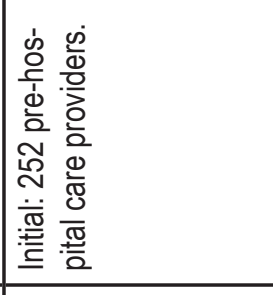 & 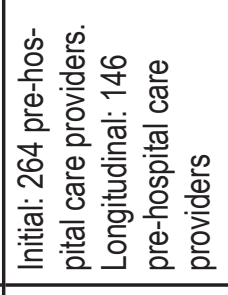 & 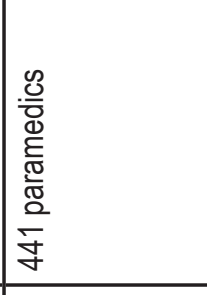 & 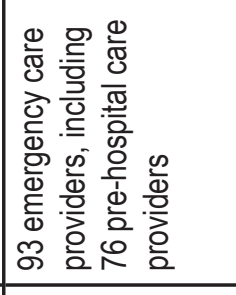 & 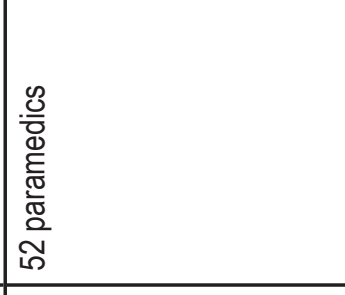 \\
\hline 호용 & 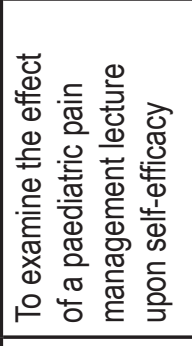 & 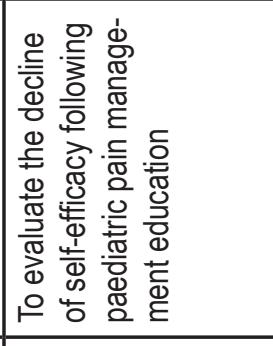 & 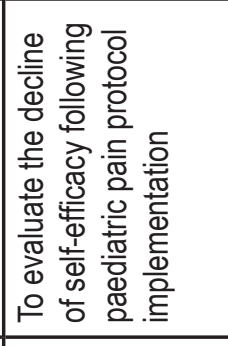 & 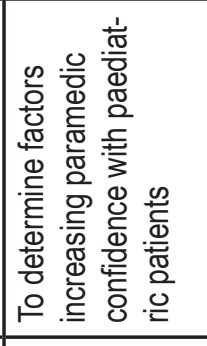 & 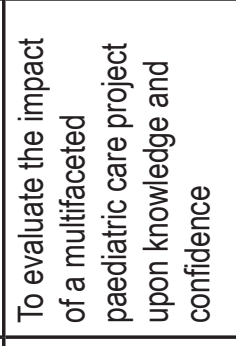 & 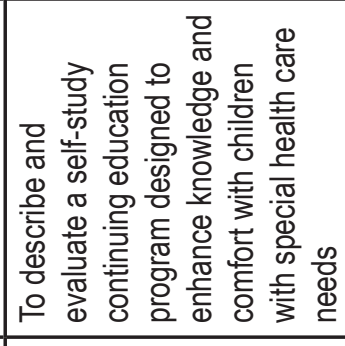 \\
\hline 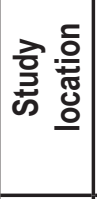 & 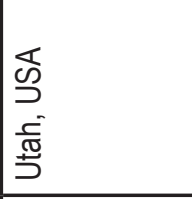 & 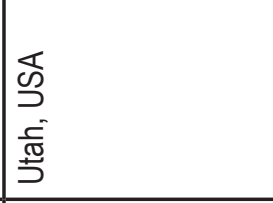 & 尔 & 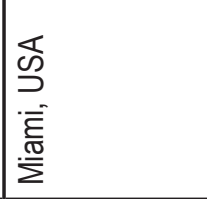 & 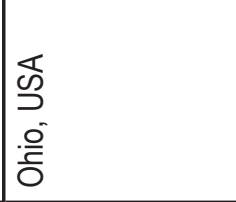 & 芯 \\
\hline 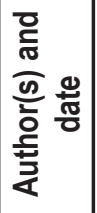 & 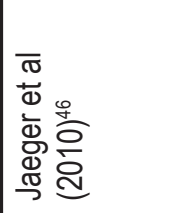 & 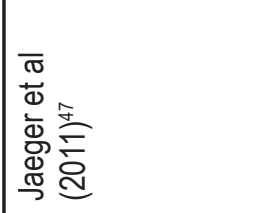 & 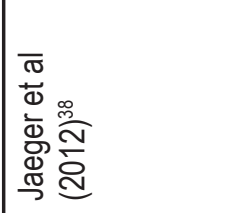 & 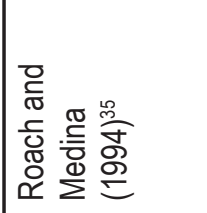 & 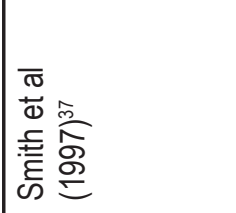 & 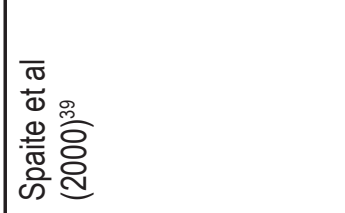 \\
\hline
\end{tabular}




\begin{tabular}{|c|c|c|c|c|c|}
\hline & 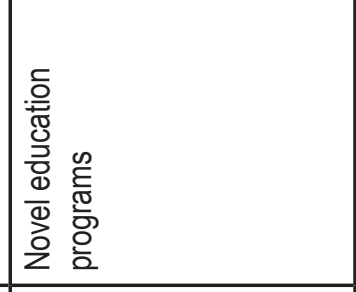 & 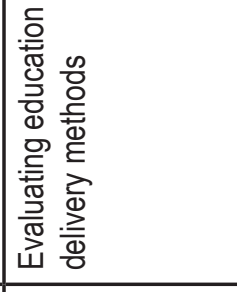 & 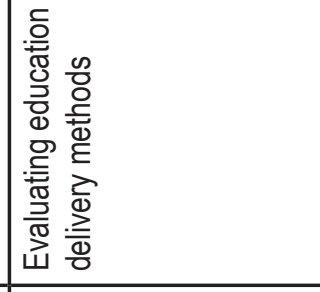 & 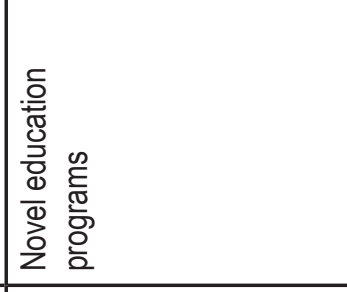 & 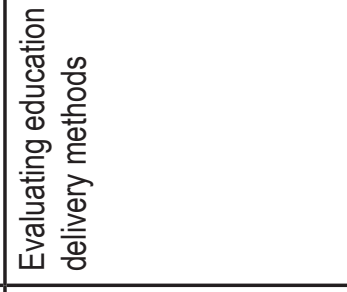 \\
\hline 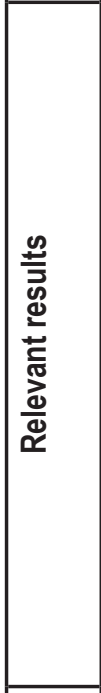 & 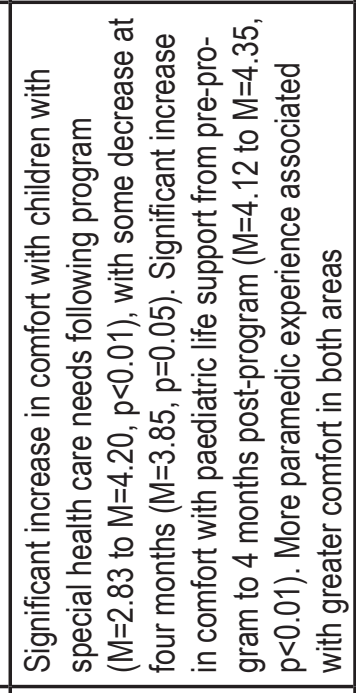 & 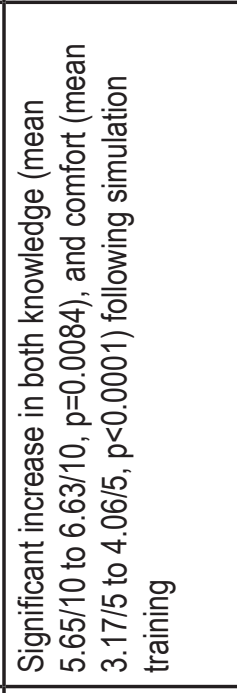 & 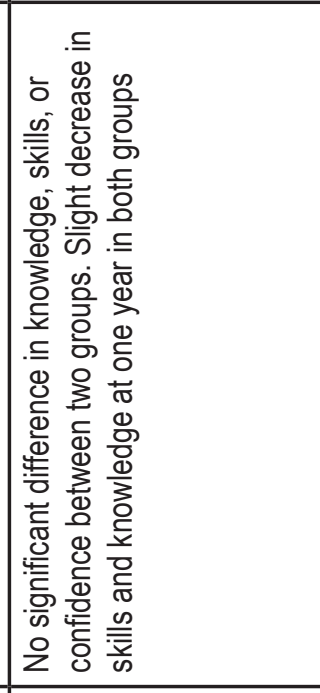 & 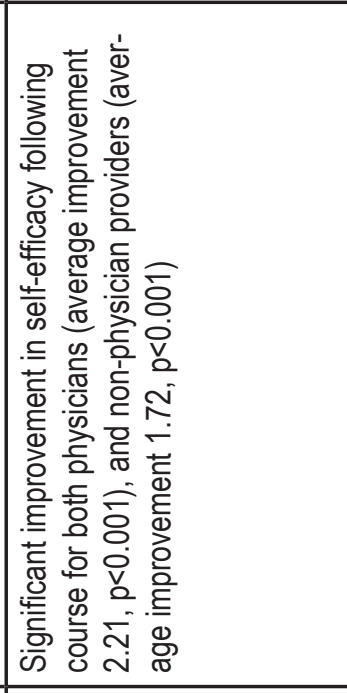 & 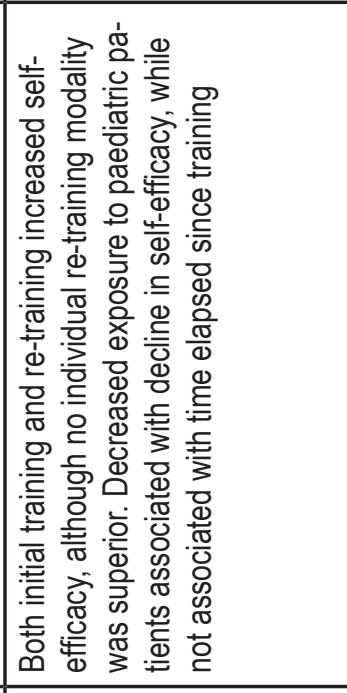 \\
\hline 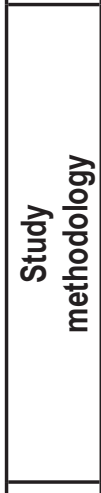 & 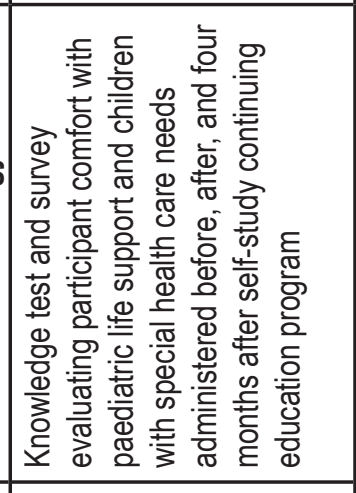 & 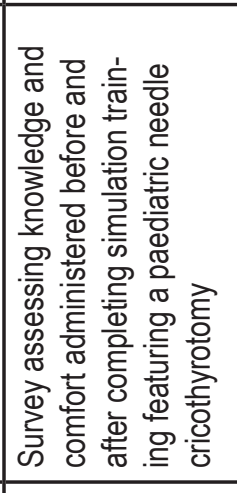 & 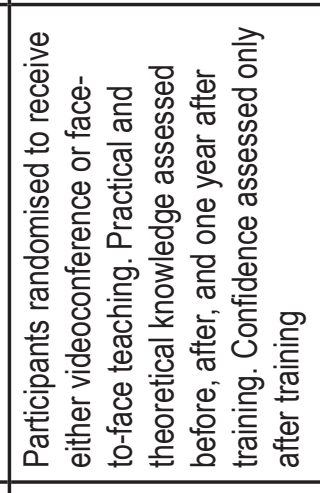 & 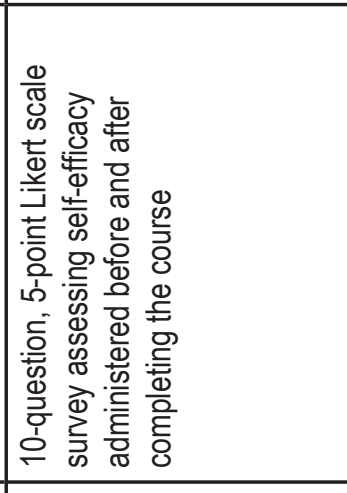 & 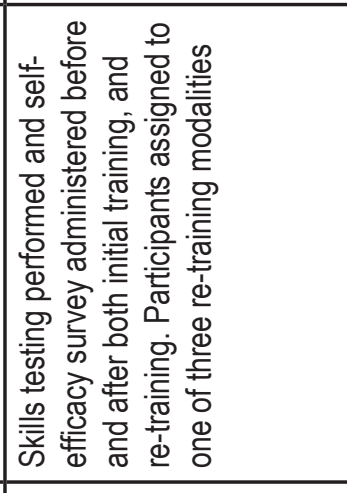 \\
\hline 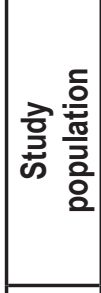 & 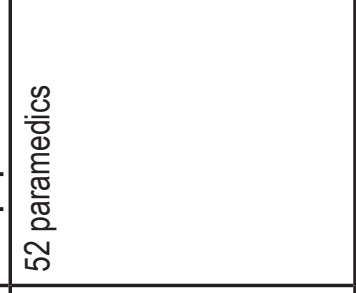 & 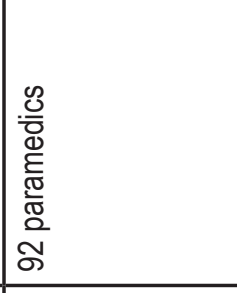 & 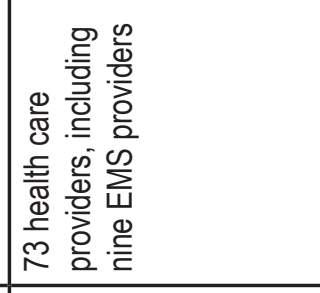 & 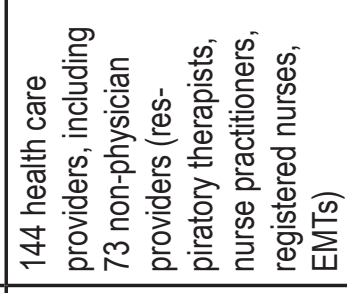 & 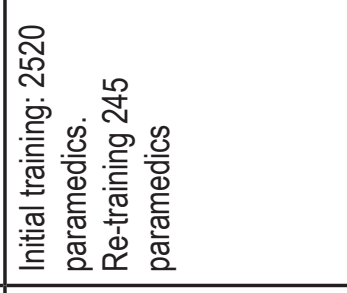 \\
\hline 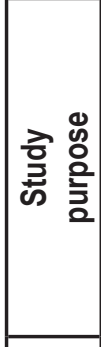 & 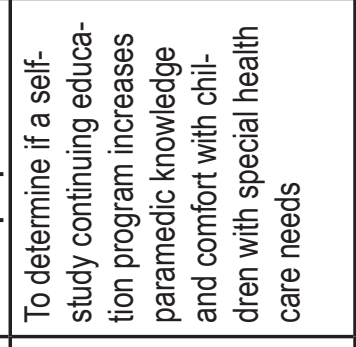 & 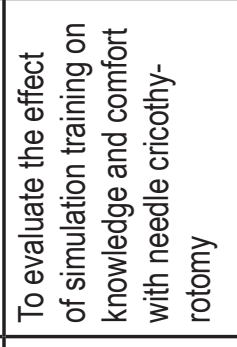 & 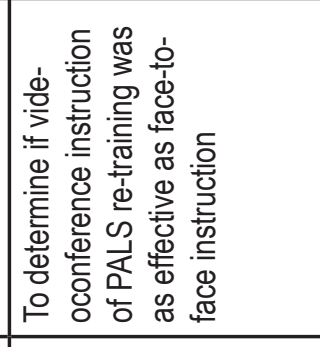 & 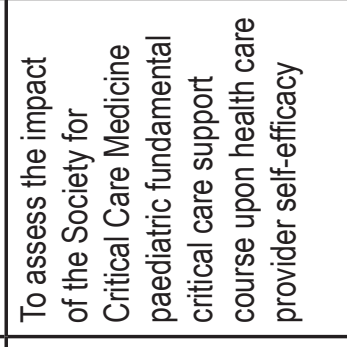 & 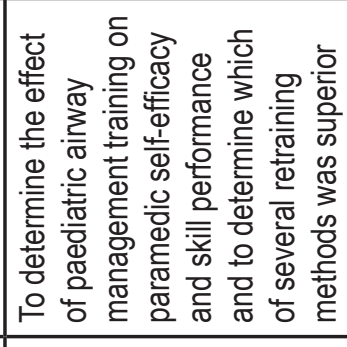 \\
\hline 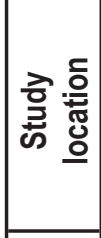 & 起 & & 昏 & & 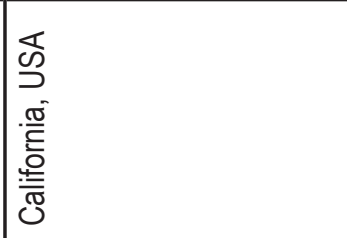 \\
\hline 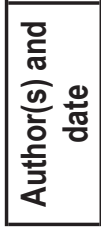 & 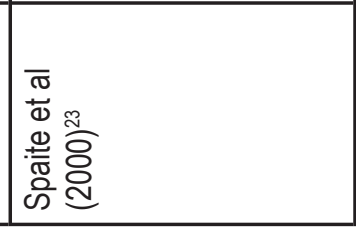 & 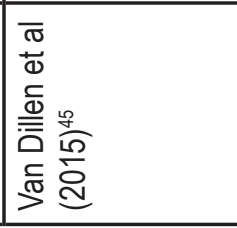 & 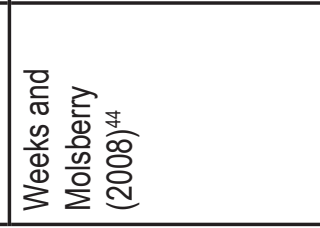 & 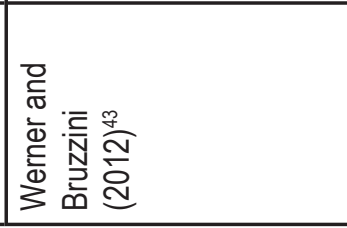 & 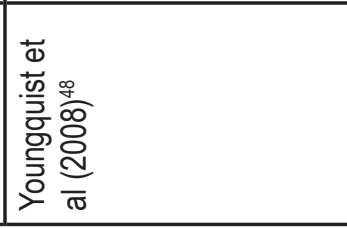 \\
\hline
\end{tabular}




\section{Results}

\section{Collating, summarising and reporting the results}

The 17 included articles were comprised of six conference abstracts, one letter to the editor and 10 journal articles. The study location of one article could not be determined; the remainder were from the USA. Years of publication ranged from 1994 to 2015. There was notable repetition of authors, with one lead author contributing three included articles and another two authors contributing two each. An iterative process of thematic analysis produced three broad themes under which included articles could be categorised:

1. Identifying educational needs and preferences $(n=5)$

2. Novel education programs $(n=8)$

3. Evaluating education delivery methods $(n=6)$.

One author performed the initial theme development and article categorisation, which was subsequently validated by a second author.

\section{Consultation (optional)}

Arksey and O'Malley's (25) scoping methodology features an optional sixth stage of consulting relevant parties external to the authors, such as experts in the area of interest or pertinent community organisations. Given the time constraints, no expert in paramedic education in paediatric care could be located, nor could any relevant community organisation. This stage was therefore omitted.

\section{Discussion}

As the subject of $4.5-13.0 \%$ of total call-outs, paediatric patients represent a reasonable minority of paramedics' workload (1-5). Previous research suggests that cases involving children can evoke anxiety and discomfort, and that resultant feelings of low confidence and self-efficacy can cause a reluctance to initiate timely appropriate treatment, possibly contributing to patient morbidity and mortality $(21,30,31)$. However, it is unknown to what extent these apprehensionproducing perceptions are held by paramedics and what educational strategies can improve them. The objective of this study was to fill this gap by performing a scoping review to examine and map the effectiveness of educational interventions on improving paramedic perceptions of caring for paediatric patients. To achieve this, included articles were categorised into three themes: identifying educational needs and preferences, because to examine effectiveness, criterion of success must first be established; novel education programs, in order to evaluate published educational interventions; and evaluating education delivery methods, to assess whether teaching methodology can influence the ability of educational interventions to improve paramedic confidence with paediatric patients.

\section{Identifying educational needs and preferences}

Both subjective focus group studies and objective surveybased studies found that paramedics are less comfortable with paediatric patients than adults $(18,32,33)$. In Fleischman et al's (32) survey of 219 pre-hospital care providers, $72 \%$ of respondents reported feeling less than 'comfortable' across 17 topics in paediatric care. Multiple studies echoed these findings, reporting that younger patient age is associated with greater discomfort $(18,32,34)$. Paramedics appear more comfortable with specific procedural skills, for example airway management, than broader tasks such as assessing a child with a long-term illness $(18,32)$.

All articles demonstrated that pre-hospital care providers desire more education in paediatric care (18,32-35). The duration and amount of training preferred however, is less clear ranging from 1 to 16 hours in duration $(32,34)$. Simulation appears to be the preferred type of education, as it can replicate both the clinical and emotional aspects of caring for paediatric patients $(18,32,33)$. Two articles identified exposure to children as a beneficial form of education, whether through parenthood or placements in local schools, paediatrician clinics or paediatric hospitals $(18,35)$. Perspectives of online training also varied between studies. While Breon et al's (18) subjective information suggested online training was preferred, only $2 \%$ of respondents to Fleischman et al's (32) survey preferred internet-based training.

All articles examined barriers to paediatric education, the most common being cost, time and quality of training (18,32-35). Costs associated with both the education itself and travel to education sites were significant barriers for many, the latter of which could be overcome with online education $(18,32,34)$. Despite Roach and Medina (35) recommending in 1994 that the paediatric advanced life support course be specifically adapted for a pre-hospital provider audience, it appears this has not occurred, with many in Breon et al's (18) 2011 study stating that pre-established courses such as PALS were not relevant to pre-hospital care.

\section{Novel education programs}

Articles categorised under this theme evaluated how effectively education programs, many of which were original and new, improved paramedic perceptions of providing care for paediatric patients. In their conference abstract, Calhoun et al (36) describe a novel simulation-based mobile outreach. Although the results of 54 participants (comprising only 15 EMTs) are presented in aggregate, comfort levels significantly increased in all domains $(p<0.001)$, suggesting this mobile simulation program could be an effective way of educating rural paramedics (36). Smith et al (37) developed a multifaceted program focussed on rural practitioners spanning initial and ongoing training, equipment acquisition and clinical 
guideline development. Interestingly, despite its extensive nature, this program did not have a more noticeable positive effect on paramedic confidence when compared with other included articles. For example, although not described in as much detail, following Jaeger et al's (38) simpler paediatric pain protocol, paramedic self-efficacy at paediatric pain assessment and management significantly improved for all patient age groups (all $p<0.05$ ). This could be reflective of Smith's more rigorous methodology and broader scope of evaluation, or it could suggest that wide-reaching complexity does not necessarily result in proportionally greater improvements to paramedic confidence (37).

Two included articles published in the same year with almost identical authors both describe a novel program designed to increase paramedic comfort when working with children with special health care needs $(23,39)$. It is likely that these articles are both evaluating the same education program, and hence they are discussed together. In both studies, following a comprehensive process of literature review and stakeholder consultation, Spaite et al $(23,39)$ developed a self-study program featuring videos, a self-study manual, quizzes, case scenarios and a 2-hour practical session involving feedback from experts. This program had remarkably positive results; the aggregate comfort score regarding specialised management skills improved from 2.83 to $4.20(p<0.01)$ on a 5-point Likert scale, while confidence with standard paediatric advanced life support skills also significantly improved (4.12 to $4.35, p<0.01$ ), suggesting specialised education programs may have flow-on effects to other areas of paediatric care (39). The success of this program demonstrates the importance of thorough consultation during curriculum development. While the self-study format appears effective, participants perceived the practical skills evaluation as the most useful component (rated 4 or 5 out of 5 for usefulness by $81.4 \%$ of respondents), highlighting the continued importance of some face-to-face teaching (39).

Pre-established programs such as the PALS and advanced paediatric life support (APLS) courses have been shown to increase confidence with paediatric care in other health care professions, although the efficacy within paramedic practice remains unclear $(40,41)$. In their letter to the editor, Roach and Medina (35) found that paramedics who had completed a PALS course had higher perceived comfort (3.6 vs. 3.2 on a 5-point Likert scale, $p=0.0001)$ and skill (3.97 vs. $3.48, p=0.0001$ ) with paediatric care than those who had not. However, PALS has undergone numerous curriculum adjustments since this article's publication in 1994, limiting applicability to a modern context. More recently and although not the primary objective, Gerard et al (42) found that the PALS course increased aggregate mean self-confidence among 42 health care practitioners (comprising 15 paramedics) from 3.57 to 4.24 on a 5-point Likert scale, although no statistical significance data were provided. Werner and Bruzzini (43) similarly found that the less-renowned Society for Critical Care Medicine Paediatric Fundamental Critical Care Support course improved feelings of preparedness for paediatric care among the non-physician group, indicating prefabricated programs may be effective in a paramedic context, although more research is needed.

\section{Evaluating education delivery methods}

Articles categorised under this theme primarily evaluated the way in which education programs were delivered, often comparing different program modalities.

Online training has many theoretical benefits; reduced instructor related costs, ensuring material is taught in a consistent manner, reduced travel and onsite time and enabling students to review material at a convenient time at their own pace $(42,44)$. Gerard et al $(42)$ sought to validate these benefits by comparing the effects of a web-based PALS course with a traditionally taught face-to-face course. Both delivery methods resulted in comparable improvements in mean paediatric care self-confidence across a 39-item, 5-point Likert scale survey (web-based 3.77-4.28, difference 0.51; traditional 3.57-4.24, difference 0.67) (42). Weeks and Molsberry (44) reached similar conclusions in a planned noninferiority trial in which 73 health care professionals were randomised to receive identical PALS re-certification education either via web-based videoconference or traditional face-toface teaching. There was no significant difference in postcourse aggregated average paediatric confidence scores (webbased 8.3 , traditional $8.5, p=0.598$ ) (44). Although paramedics comprised a significant proportion of the study populations in both of these articles, data were presented in aggregate from a diverse population of health care professions, which may limit applicability to exclusively the paramedic context $(42,44)$.

In the only included article evaluating simulation, Van Dillen et al's (45) conference abstract found that paediatric airway simulation significantly improved mean comfort scores with paediatric needle cricothyrotomy $(3.17-4.06, p<0.0001)$. This absence of literature assessing simulation is curious given its aforementioned popularity as a teaching methodology $(18,32)$. Jaeger et al (46) showed that traditional teaching remains effective, demonstrating significant improvements in paediatric pain management self-efficacy among paramedics following a 1-hour lecture. Despite this, the most effective education methodology remains to be definitively established.

\section{Implications for research}

Throughout all included studies there was no standardised validated tool to measure paramedic perceptions or confidence when dealing with paediatric patients. Development of such a tool may enable better comparison between future studies, as well as simplifying implementation of research protocols. The absence of any included studies from outside the USA suggests a need to conduct research elsewhere. There was a curious lack of studies evaluating simulation, especially considering its popularity, and future research could further explore simulation as an educational methodology. 
Despite being regarded as somewhat of a mainstay of paediatric pre-hospital education, evidence surrounding preestablished programs such as PALS and APLS is divided and often out dated given these programs rapidly evolving curricula. Comprehensive evaluation of such programs within a modern context and exclusively pre-hospital population could assist in determining efficacy and what, if any, modifications need to be made to ensure applicability to paramedic practice. The most effective educational program or teaching methodology remains unclear, and further research is required to clarify this. Of particular interest could be research that follows an education intervention throughout its lifespan; beginning with an assessment of educational needs and subsequent development of a customised program followed by consistent evaluation throughout its implementation. All included articles featured educational interventions targeting paramedics who had completed their initial training, and hence the influence of paediatric education within the pre-qualification curriculum remains unexplored.

\section{Limitations}

All but one of the included articles was from the USA, with the location of the final article unable to be determined. This limited range of available evidence may reduce the ability to generalise findings to other areas. The year of publication of included articles ranged from 1994 to 2015, with over half published before 2011 . This may limit the applicability of some articles to contemporary paramedic practice. The repetition of authors within included studies may have limited the breadth of information available, but this is reflective of the limited research within this area. The methodology of this study excluded articles not in English and did not include hand searching of journals, and hence some relevant results may have been omitted. The focus of scoping reviews is on mapping of key concepts and the evidence supporting a research domain of interest, and hence no appraisal of the research methodology of included articles was performed $(25,26)$.

\section{Conclusion}

Paramedics desire more paediatric training, particularly simulation and exposure to children. However, while various paediatric education programs have been developed there is insufficient evidence to demonstrate superior effectiveness of any particular program. More research evaluating the benefits of simulation as an educational methodology to improve paramedic perceptions when dealing with paediatric patients is required. The efficacy and applicability of pre-established programs such as APLS and PALS remains unclear, and further research is required to evaluate such programs within a modern pre-hospital context. While the most effective education delivery method is unclear, is seems web-based learning can be as effective as traditional teaching. Further research is needed to identify and develop the most effective educational interventions to improve paramedic confidence with paediatric patients.

\section{Conflict of interest}

The authors declare no competing interests. The authors of this paper have completed the ICMJE conflict of interest statement.

\section{References}

1. Kloot K, Salzman S, Kilpatrick S, Baker T, Brumby SA. Initial destination hospital of paediatric prehospital patients in rural Victoria. Emerg Med Australas 2016;28:205-10.

2. Harve H, Salmi H, Rahiala E, Pohjalainen P, Kuisma M. Out] of thospital paediatric emergencies: a prospective, populationbased study. Acta Anaesthesiol Scand 2016;60:360-9.

3. Eich C, Russo SG, Heuer JF, et al. Characteristics of outof-hospital paediatric emergencies attended by ambulanceand helicopter-based emergency physicians. Resuscitation 2009;80:888-92.

4. Drayna PC, Browne LR, Guse CE, Brousseau DC, Lerner EB. Prehospital pediatric care: opportunities for training, treatment, and research. Prehosp Emerg Care 2015;19:4417.

5. Shah MN, Cushman JT, Davis CO, Bazarian JJ, Auinger $\mathrm{P}$, Friedman B. The epidemiology of emergency medical services use by children: an analysis of the National Hospital Ambulatory Medical Care Survey. ibid. 2008;12:269-76.

6. Australian Institute of Health and Welfare. A picture of Australia's children 2012. Cat. no. PHE 167 Canberra: AlHW; 2012. Available at: www.aihw.gov.au/WorkArea/ DownloadAsset.aspx?id=10737423340

7. Centers for Disease Control and Prevention. 10 leading causes of death by age group, United States - 2014: National Centre for Injury Prevention and Control, CDC; 2014. Available at: www.cdc.gov/injury/images/lc-charts/leading_ causes_of_death_age_group_2014_1050w760h.gif

8. World Health Organization. Child and adolescent injury prevention: a global call to action. Geneva, Switzerland, 2005. Available at: http://apps.who.int/iris/ bitstream/10665/43279/1/9241593415_eng.pdf

9. Stevens SL, Alexander JL. The impact of training and experience on EMS providers' feelings toward pediatric emergencies in a rural state. Pediatr Emerg Care 2005;21:12-7.

10. Guise J, Meckler G, O'Brien K, et al. Patient safety perceptions in pediatric out-of-hospital emergency care: Children's Safety Initiative. J Pediatr 2015;167:1143-8.

11. Cushman JT, Fairbanks RJ, O'Gara KG, et al. Ambulance personnel perceptions of near misses and adverse events in pediatric patients. Prehosp Emerg Care 2010;14:477-84.

12. Cottrell EK, O'Brien K, Curry M, et al. Understanding safety in prehospital emergency medical services for children. ibid. 2014;18:350-8. 


\section{References (continued)}

13. Institute of Medicine. Emergency care for children: growing pains Washington D.C., U.S.A.: National Academies Press; 2007. Available at: www.nap.edu/catalog/11655/emergencycare-for-children-growing-pains

14. Dyson K, Bray J, Smith K, Bernard S, Straney L, Finn J. Paramedic exposure to out-of-hospital cardiac arrest is rare and declining in Victoria, Australia. Resuscitation 2015;89:93-8.

15. Hoyle Jr JD, Sleight D, Henry R, Chassee T, Fales B, Mavis B. Pediatric prehospital medication dosing errors: a mixedmethods study. Prehosp Emerg Care 2016;20:117-24.

16. Miller DR, Kalinowski EJ, Wood D. Pediatric continuing education for EMTs: recommendations for content, method, and frequency. Pediatr Emerg Care 2004;20:269-72.

17. Hansen M, Meckler G, Dickinson C, et al. Children's Safety Initiative: a national assessment of pediatric educational needs among emergency medical services providers. Prehosp Emerg Care 2015;19:287-91.

18. Breon A, Yarris L, Law J, Meckler G. Determining the paediatric educational needs of prehospital providers: part 1. Journal of Paramedic Practice 2011;3:450-6.

19. Ngo TL, Belli K, Shah MI. EMSC program manager survey on education of prehospital providers. Prehosp Emerg Care 2014;:18:424-8.

20. Eburn M, Bendall J. The provision of Ambulance services in Australia: a legal argument for the national registration of paramedics. Australasian Journal of Paramedicine 2010;8:19.

21. Reavley P. The challenges of pre-hospital paediatric trauma care. Journal of Paramedic Practice 2014;6:18-20.

22. O'Meara P. Paramedics marching toward professionalism. Australasian Journal of Paramedicine 2009;7:1-5.

23. Spaite DW, Karriker KJ, Seng M, et al. Increasing paramedics' comfort and knowledge about children with special health care needs. Am J Emerg Med 2000;18:74752.

24. Armstrong R, Hall BJ, Doyle J, Waters E. 'Scoping the scope' of a Cochrane review. J Public Health 2011;33:147-50.

25. Arksey H, O'Malley L. Scoping studies: towards a methodological framework. Int J Soc Res Methodol 2005;8:19-32.

26. Daudt HML, van Mossel C, Scott SJ. Enhancing the scoping study methodology: a large, inter-professional team's experience with Arksey and O'Malley's framework. BMC Med Res Methodol 2013;13(48).

27. Levac D, Colquhuon H, O'Brien KK. Scoping studies: advancing the methodology. Implement Sci 2010;5(69).

28. The Joanna Briggs Institute. The Joanna Briggs Institute Reviewers' Manual 2015: Methodology for JBI Scoping Reviews. Adelaide, Australia: The Joanna Briggs Institute; 2015.

29. Burgess S, Smith E, Piper S, Archer F. The development of an updated prehospital search filter for the Cochrane Library: Prehospital Search Filter Version 2.0 Australasian Journal of Paramedicine 2010;8:1-4.

30. Maibach E, Schieber R, Carroll M. Self-efficacy in pediatric resuscitation: Implications for education and performance. Pediatr 1996;97:94-9.

31. Turner NM, Lukkassen I, Bakker N, Draaisma JMT, ten Cate $\mathrm{O}$. The effect of the APLS-course on self-efficacy and its relationship to behavioural decisions in paediatric resuscitation. Resuscitation 2009;80:913-8.

32. Fleischman RJ, Yarris LM, Curry MT, Yuen SC, Breon AR, Meckler GD. Pediatric educational needs assessment for urban and rural emergency medical technicians. Pediatr Emerg Care 2011;27:1130-5.

33. Breon A, Yarris L, Law J, Meckler G. Determining the paediatric educational needs of prehospital providers: part 2 . Journal of Paramedic Practice 2011;3:510-4.

34. Glaeser PW, Linzer J, Tunik MG, Henderson DP, Ball J. Survey of nationally registered emergency medical services providers. Pediatr Educ Ann Emerg Med 2000;36:33-8.

35. Roach CL, Medina FA. Paramedic comfort level with children in medical and trauma emergencies: does the PALS course make a difference? Am J Emerg Med 1994;12:260-2.

36. Calhoun A, Porter M, Maus-Granholm P, Montgomery V, Orman $\mathrm{K}$. Enhancing the emergency care of critically ill children using mobile simulation technology. Crit Care Med 2012:40:153.

37. Smith GA, Thompson JD, Shields BJ, Manley LK, Haley KJ. Evaluation of a model for improving emergency medical and trauma services for children in rural areas. Ann Emerg Med 1997;29:504-10.

38. Jaeger A, Holsti M, Dudley N, Sheng X, Gurley K, Adelgais K. EMS provider self-efficacy retention after pediatric pain protocol implementation. Acad Emerg Med 2012;19:S86.

39. Spaite DW, Karriker KJ, Seng M, et al. Training paramedics: emergency care for children with special health care needs. Prehosp Emerg Care 2000;4:178-85.

40. Turner NM, Dierselhuis MP, Draaisma JMT, ten Cate O. The effect of the advanced paediatric life support course on perceived self-efficacy and use of resuscitation skills. Resuscitation 2007;73:430-6.

41. Dhingra P, Ngeth P, Prak M, Ung S. Assessment of the effect of advanced paediatric life support training on level of self-perceived preparedness among health-care workers in Cambodia. Emerg Med Australas 2012;24:329-35.

42. Gerard JM, Scalzo AJ, Laffey SP, Sinks G, Fendya D, Seratti P. Evaluation of a novel web-based pediatric advanced life support course. Arch Pediatr Adolesc Med 2006;160:649-55.

43. Werner J, Bruzzini D. PFCCS improves perceived selfefficacy in a variety of providers. Crit Care Med 2012;40:155.

44. Weeks DL, Molsberry DM. Pediatric advanced life support re-training by videoconferencing compared to face-to-face instruction: a planned non-inferiority trial. Resuscitation 2008;79:109-17. 


\section{References (continued)}

45. Van Dillen C, Fitzpatrick D, Rowe J, Weeks E. effect of simulation training on prehospital provider competence and knowledge with needle cricothyrotomy. Acad Emerg Med 2015;22:S245-6.

46. Jaeger A, Holsti M, Dudley N, Sheng X, Gurley K, Adelgais $K$. Pain management education effect on EMS provider selfefficacy. ibid. 2010;17:S173-4.

\section{Appendix 1. Search strategy}

Component one: pre-hospital search filter. From Burgess S, Smith E, Piper S, Archer F. The development of an updated prehospital search filter for the Cochrane Library: Pre-hospital Search Filter Version 2.0 Journal of Emergency Primary Health Care. 2010;8:1-4.

1. [MeSH] 'Emergency Medical Services'

2. [MeSH] 'Emergency Medical Technicians'

3. [MeSH] 'Emergency Treatment'

4. [MeSH] 'Emergency Medicine'

5. [MeSH] 'Ambulances'

6. MeSH] 'First Aid'

7. [MeSH] 'Military Medicine'

8. prehospital

9. pre-hospital

10. paramedic*

11. ambulance*

12. out-of-hospital

13. out of hospital

14. ems

15. emt

16. emergency services

17. emergency medical service*

18. emergency technician*

19. emergency practitioner

20. emergency dispatch*

21. emergency despatch ${ }^{*}$

22. first responder*

23. public access defibrillation

24. emergency rescue

25 . emergency resus ${ }^{*}$

26. emergency triage

27. advanced life support

28. community support co-ordinator

29. community support coordinator

30. emergency care practitioner

31. extended care practitioner

32. physician assistant
47. Jaeger A, Holsti M, Dudley N, Sheng X, Gurley K, Adelgais K. Prehospital provider self-efficacy decline after pediatric pain management education. Acad Emerg Med 2011;: S116.

48. Youngquist ST, Henderson DP, Gausche-Hill M, Goodrich SM, Poore PD, Lewis RJ. Paramedic self-efficacy and skill retention in pediatric airway management. ibid. 2008;15:1295-303.

33. 1 OR 2 OR 3 OR 4 OR 5 OR 6 OR 7 OR 8 OR 9 OR 10 OR 11 OR 12 OR 13 OR 14 OR 15 OR 16 OR 17 OR 18 OR 19 OR 20 OR 21 OR 22 OR 23 OR 24 OR 25 OR 26 OR 27 OR 28 OR 29 OR 30 OR 31 OR 32

Component two: paediatric

1. [MeSH] 'Child'

2. [MeSH] 'Infant'

3. [MeSH] 'Adolescence'

4. [MeSH] 'Pediatrics'

5. pediatric*

6. child*

7. paediatric*

8. 1 OR 2 OR 3 OR 4 OR 5 OR 6 OR 7

Component three: educational interventions

1. [MeSH] 'Education'

2. [MeSH] 'Teaching'

3. training in some databases as [MeSH] 'Inservice Training'

4. 1 OR 2 OR 3

Component four: confidence

1. [MeSH] "Self-Efficacy"

2. [MeSH] 'Anxiety'

3. [MeSH] 'Anticipatory Anxiety'

4. [MeSH] 'Emotions'

5. [MeSH] 'Courage' (only available in some databases)

6. comfort $^{*}$

7. opinion*

8. confiden*

9. 'knowledge gap"'

10. prepar* NOT disaster

11. 1 OR 2 OR 3 OR 4 OR 5 OR 6 OR 7 OR 8 OR 9

Final strategy

Combine the final line of each component with the Boolean operator "AND" 


\section{Appendix 2. Inclusion and exclusion criteria}

Inclusion criteria:

1. Population

a. Pre-hospital care providers of any skill level

b. Paramedic students - included as paramedics are often exposed to educational interventions whilst students.

2. Concept

a. Appraisal of paramedic perceptions of, or confidence when dealing with paediatric patients

b. Description or evaluation of educational intervention(s) aimed at improving paramedic confidence when dealing with paediatric patients. Educational interventions became defined as: anything featuring teaching, training, or practice supervision.
3. Context

a. Care providers practising in a pre-hospital setting

b. Relating to paediatric patients. Paediatric was defined as less than or equal to 18 years of age.

Exclusion criteria:

1. Articles not written in English due to financial and time restraints preventing translation.

2. Examination of only clinical skills without any reference to paramedic perceptions of, or confidence when dealing with paediatric patients.

3. Study population of physicians or nurses, even if practising in the pre-hospital field. This was established to supplement the population inclusion criteria and ensure only paramedics were included, rather than retrieval nurses and physicians. 